\title{
Analysis of circulating adipokines in patients newly diagnosed with solid cancer: Associations with measures of adiposity and tumor characteristics
}

\author{
NEHAD AYOUB, MOHAMMAD ALKHATATBEH, MALAK JIBREEL and MERA ABABNEH
}

Department of Clinical Pharmacy, Faculty of Pharmacy, Jordan University of Science and Technology, Irbid 22110, Jordan

Received July 21, 2016; Accepted November 29, 2016

DOI: $10.3892 / \mathrm{ol} .2017 .5670$

\begin{abstract}
The development and progression of cancer is a complex and multifactorial process and the global prevalence of obesity is markedly increasing. A number of studies have made an association between obesity and increased rates of epithelial tumors. Obesity is associated with altered adipokine levels, potentially contributing to the process of tumor development and metastasis. In the current study, the associations between circulating adipokines and measures of adiposity and tumor characteristics among patients diagnosed with solid malignancies were examined at the time of presentation, and following the administration of chemotherapy. A total of 30 patients with cancer and matched healthy controls were enrolled in the present study. Plasma adipokine levels of hepatocyte growth factor (HGF), adiponectin and leptin were determined using commercially available ELISA kits. At baseline, plasma HGF, adiponectin and leptin levels were not significantly different between patients with cancer and the healthy controls. Circulating HGF levels were significantly associated with the stage of cancer at diagnosis $(\mathrm{P}=0.044)$, but lacked a significant association with lymph node status $(\mathrm{P}=0.194)$. Plasma adiponectin and leptin levels were not significantly associated with tumor characteristics at the time of diagnosis. Only leptin was positively correlated with the body mass index of patients with cancer $(\mathrm{P}<0.001)$. No significant correlations were detected between the evaluated adipokines and measures of visceral obesity, as determined by waist circumference and the waist-hip ratio at presentation. Following administration of chemotherapy, adiponectin was the only adipokine evaluated in the current study that
\end{abstract}

Correspondence to: Dr Nehad Ayoub, Department of Clinical Pharmacy, Faculty of Pharmacy, Jordan University of Science and Technology, P.O. Box 3030, Ar Ramtha, Irbid 22110, Jordan E-mail:nmayoub@just.edu.jo

Abbreviations: HGF, hepatocyte growth factor; BMI, body mass index

Key words: solid tumors, adipokines, obesity, hepatocyte growth factor, adiponectin, leptin exhibited a significant difference, when compared with baseline plasma levels $(\mathrm{P}=0.013)$, and a significant positive correlation between baseline and follow-up circulating levels $(\mathrm{P}=0.002)$ among patients with cancer. In addition, there were no significant inter-correlations between circulating adipokines at baseline level and during follow-up in patients with cancer. Collectively, the findings of the current study suggest a lack of diagnostic roles for the adipokines investigated and no significant association with measures of adiposity. Adiponectin may be a potential adipokine to measure in patients with cancer, in order to further assess its prognostic and predictive potential.

\section{Introduction}

Cancer is a major public health problem and is a leading cause of mortality worldwide (1). Annual cancer cases are predicted to rise from 14 million in 2012 to 22 million within the next two decades (2). In Jordan, there has been a notable increase in the incidence and burden of cancer and, according to the most recent report from the Jordan National Cancer Registry, the number of new cancer cases diagnosed among Jordanians increased by $\sim 46 \%$ from 2000-2012 (3). The most common types of cancer among Jordanian patients were breast, colorectal, lung, lymphoma and urinary bladder cancer (3). At present, epidemiological and experimental evidence suggests an association between obesity and increased risk of several types of cancer $(4,5)$.

Obesity is defined as an excess accumulation of adipose tissue in the body (6). According to the World Health Organization (WHO), the accepted classification of obesity for epidemiological purposes defines overweight individuals as having a body mass index (BMI) of $\geq 25 \mathrm{~kg} / \mathrm{m}^{2}$ and obese subjects as having BMI of $\geq 30$ (7). Globally, $>1.9$ billion adults were overweight and, of these, $>600$ million were obese in 2014 (6). At present, 5\% of cancer cases may be directly associated with patients being overweight (8). In addition to increasing the likelihood of developing cancer, obesity has also been associated with increased cancer mortality (4). A number of underlying mechanisms may mediate obesity-induced carcinogenesis, including insulin resistance, increased inflammation and altered adipokine secretion $(5,8,9)$.

Adiponectin and leptin are adipokines that are exclusively secreted by adipose tissue (10). Obesity is associated 
with reduced circulating levels of secreted adiponectin and increased circulating levels of leptin (11). A number of studies have focused on major adipokines in association with the risk of solid tumors (12-14). However, few studies have evaluated the correlations between circulating adipokine levels and the tumor characteristics of patients at the time of diagnosis. Hepatocyte growth factor (HGF) and its tyrosine kinase receptor, c-Met, have been associated with the majority of human cancer types, and their expression levels frequently correlate with poor prognosis and the presence of metastases $(15,16)$. Previous studies have revealed that HGF is secreted by adipose tissue and that its circulating levels are elevated in obese individuals $(9,17)$. Although a number of studies have demonstrated that HGF is an important factor in solid tumor progression and metastasis, the interplay between HGF and common adipokines, in terms of adiposity and the tumor characteristics of patients with cancer at the time of presentation, remains to be elucidated.

Obesity contributes to an increased risk of cancer development, and it is established that adiposity alters the levels of adipokines secreted, which promotes cancer proliferation and dissemination (18). However, the association between the circulating levels of adipokines in patients with cancer at the time of diagnosis has yet to be characterized. In addition, the associations between certain adipokines and measures of adiposity among patients with cancer require further study. Therefore, the purpose of the current study was to assess plasma adipokine levels in a sample of patients diagnosed with solid tumors, and to further assess changes to baseline levels following the administration of chemotherapy. The present study also assessed the correlations between the circulating levels of selected adipokines and the tumor characteristics and measures of adiposity at the time of diagnosis among patients with cancer.

\section{Materials and methods}

Patients and study design. Patients with a diagnosis of solid malignancy (described below) were recruited prospectively from the Oncology Clinic at King Abdullah University Hospital (KAUH; Ar Ramtha, Jordan) between June 2014 and December 2015. Types of solid cancers diagnosed among patients in this study included colorectal, breast, lung, testicular and gastric. The study population was composed of adult patients ( $>18$ years) who had a first-time diagnosis of solid malignancy. The diagnosis of malignancy was performed using histopathological analysis of resected tumor, biopsy or cytology specimens at the pathology department of KAUH. The stage of the diagnosed tumors was determined according to the tumor-node-metastasis classification system (19). Newly diagnosed patients with cancer who were receiving neoadjuvant chemotherapy or radiation therapy at time of the current study were not eligible to participate. The study sample was matched to healthy volunteers based on age, gender and BMI. Healthy volunteers were recruited from individuals who attended KAUH as visitors or for other purposes. All patients provided written informed consent prior to their participation in the study. The study was approved by the Institutional Review Board at KAUH and the Jordan University of Science and Technology (research number 20140057).
Data collection and tumor characteristics. All the relevant data for eligible patients was collected through a detailed review of medical records and retrieval of patient information from electronic databases at the time of the diagnosis of malignancy. Tumor data for patients was extracted from relevant pathology reports issued by KAUH. Anthropometric measurements for body weight $(\mathrm{kg})$, height $(\mathrm{cm})$, waist $(\mathrm{cm})$ and hip $(\mathrm{cm})$ circumferences were obtained for all subjects according to the WHO recommendations (20). Visceral obesity was evaluated using measurements of waist circumference and the waist-hip ratio, which was calculated by dividing the waist circumference by the hip circumference (18). BMI was calculated using the standard method, in which weight in kilograms is divided by the square of the height in meters (7). Patients were divided into groups based on their obesity classifications (7). WHO defines BMI classes as follows: Underweight $\left(<18.5 \mathrm{~kg} / \mathrm{m}^{2}\right)$, normal $\left(18.5-24.99 \mathrm{~kg} / \mathrm{m}^{2}\right)$, overweight $\left(\geq 25.00 \mathrm{~kg} / \mathrm{m}^{2}\right)$ and obese $\left(\geq 30.00 \mathrm{~kg} / \mathrm{m}^{2}\right)(7)$.

Collection and processing of biological samples. Blood samples for the studied adipokines were obtained at baseline level and after two months of chemotherapy administration for patients with cancer. Baseline blood samples were collected from eligible patients following a diagnosis of solid malignancy and prior to surgical intervention or the administration of chemotherapy or radiation. The second blood sample was collected after eight weeks of administrating of the assigned chemotherapy (21). This time point represents an adequate trial of chemotherapy, and clinical assessment of patients can be considered at this stage for future decision-making (21). The interval between the two time points of blood sampling in the current study was hypothesized to be sufficiently long for the detection of any alterations in the circulating levels of target adipokines. Blood samples for all patients were obtained by venipuncture of an antecubital vein of the forearm. Blood samples were collected in EDTA tubes (Greiner Bio-One GmbH, Kremsmünster, Austria) and were processed within two h of collection. Plasma samples were prepared by double centrifugations at $3000 \mathrm{x} g$ for $15 \mathrm{~min}$ each at room temperature (22). Plasma aliquots were stored at $-80^{\circ} \mathrm{C}$ until analysis.

Adipokine analysis. Plasma concentrations of adipokines were measured using ELISA kits (Quantikine ${ }^{\circledR}$ or DuoSet $^{\circledR}$ ELISA kits; R\&D Systems, Inc., Minneapolis, MN, USA), according to the manufacturer's protocol. Analysis was performed to measure plasma levels of human HGF (\#DHG00), adiponectin (\#DRP300), and leptin (\#DY398-05). The assay sensitivity was $40 \mathrm{pg} / \mathrm{ml}$ for HGF, $0.891 \mathrm{ng} / \mathrm{ml}$ for adiponectin and $31.2 \mathrm{pg} / \mathrm{ml}$ for leptin. Plasma samples were diluted 1:200 for the quantification of adiponectin. A standard concentration curve was produced for each ELISA plate with the manufacturer's control solution and used to calculate plasma concentrations in the samples assayed. Plasma samples were thawed at $37^{\circ} \mathrm{C}$ immediately prior to analysis. During sample analysis, researchers were blinded to the sample anthropometric and clinical data. All samples were analyzed in duplicate, and a fresh aliquot was used for each analysis with no prior freeze-thaw cycles. 
Statistical analysis. Data analysis was performed using SPSS version 21.0 (IBM SPSS, Armonk, NY, USA). As the continuous variables were not normally distributed, non-parametric statistical tests were applied. Continuous variables were presented as medians and interquartile ranges (IQRs) expressed by the 25 th and 75 th percentiles. Categorical variables were presented as the frequency and percentages. In order to compare between two groups, the Mann-Whitney $\mathrm{U}$ test was applied for independent groups and the Wilcoxon signed rank test was used for paired data. Kruskal-Wallis analysis of variance was used to compare multiple independent groups when indicated. To compare categorical variables between groups, Pearson's $\chi^{2}$ test of independence was used. To assess correlations between continuous variables, Spearman's correlation test was applied. All P-values were two-sided at a value of 0.05 . Boxplots are non-parametric representations of continuous data. In this type of graph, the median is presented as a vertical line and the IQR spans from the lower to the upper edge of the box. The whiskers above and below the box represent non-outlying maximum and minimum data points, respectively (23). $\mathrm{P}<0.05$ was considered to indicate a statistically significant difference.

\section{Results}

Study population. A total of 32 plasma samples were collected from patients with cancer at the baseline level, and 2 patients subsequently requested to withdraw their consent and not participate in the study. Therefore, 30 samples were available for final analysis. In total, 16 patients with cancer provided blood samples after 8 weeks of chemotherapy (16/30). Certain patients were unable to provide follow-up samples due to succumbing to mortality prior to the assigned time for the second blood withdrawal and/or due to alterations in scheduled chemotherapeutic treatments for various reasons.

The demographic, anthropometric and adipokine characteristics of the study population. The demographic and anthropometric characteristics for patients with cancer $(n=30)$ and their matched healthy controls $(n=30)$ are presented in Table I. The median age for patients with cancer was 47 years (IQR, 38.8-57.5), and the median age for healthy controls was 45 years (IQR, 38.8-56.5). The median BMI for patients with cancer was $26.5 \mathrm{~kg} / \mathrm{m}^{2}$ (IQR, 22.4-31.4). The median waist circumference and waist-hip ratio among cancer patients at the time of diagnosis were $95 \mathrm{~cm}$ and 0.91 , respectively. There were no significant differences in all reported anthropometric measurements of general and abdominal obesity between patients with cancer and healthy controls (Table I). In addition, there were no significant differences in the baseline circulating plasma concentrations of HGF, adiponectin and leptin between patients with cancer and healthy controls (Table I).

Analysis of baseline adipokines for patients with cancer. Table II presents an analysis of baseline adipokine levels and tumor characteristics for patients with cancer. The majority of patients had a diagnosis of colorectal carcinoma (43.3\%). Other patients $(33.3 \%)$ were diagnosed with breast cancer. Other types of solid malignancies diagnosed included lung, gastric and testicular cancer. In total, $70 \%$ of patients had advanced-stage disease (stage III and IV) at the time of diagnosis, and the majority of patients had lymph-node involvement (75\%) and received surgical intervention following the diagnosis of malignancy (80\%). All patients received adjuvant chemotherapy, whereas only four patients received adjuvant radiotherapy. An analysis of baseline adipokine levels among patients with cancer revealed a significant difference in leptin plasma levels according to the type of carcinoma diagnosed ( $\mathrm{P}=0.004$; Table II). The median plasma levels of HGF and adiponectin were not significantly different among the tumor types analyzed in this study $(\mathrm{P}=0.840$ and $\mathrm{P}=0.483$ for $\mathrm{HGF}$ and adiponectin, respectively). Baseline HGF plasma levels differed significantly between patients diagnosed with early-stage disease, as compared with advanced-stage disease $(\mathrm{P}=0.044)$. The median HGF plasma levels were higher for patients who presented with advanced-stage disease (median, 797.30; IQR, 628.40-958.67 pg/ml) compared with early-stage disease (median, 674.90; IQR, 493.68-742.90 pg/ml). Furthermore, baseline plasma levels of HGF were significantly lower in patients receiving surgical treatment compared with those who were not candidates for surgical interventions $(\mathrm{P}=0.038)$. There were no significant differences in baseline adipokine plasma levels between patients with cancer who had lymph-node involvement at the time of diagnosis, and those who had lymph-node negative disease (Table II).

Correlation analysis of baseline adipokine levels with anthropometric parameters of patients with cancer. The correlations between baseline adipokine levels and anthropometric measurements among patients with cancer are presented in Table III. There was a significant positive correlation between plasma HGF levels and age at diagnosis $(\rho=0.432 ; P=0.017)$. In addition, a significant positive correlation was detected between plasma leptin levels and the BMI of patients at the time of diagnosis $(\rho=0.657 ; \mathrm{P}<0.001$; Table III). However, baseline HGF and adiponectin plasma concentrations were not significantly associated with the BMI of patients (Table III). Plasma HGF, adiponectin and leptin levels were not significantly correlated with waist circumference or waist-hip ratio among patients with cancer at the baseline level (Table III). Additional multiple comparison analysis revealed a significant difference in the median leptin plasma concentration among the various BMI categories of patients with cancer ( $\mathrm{P}=0.002$; Fig. 1). In this regard, median plasma levels of leptin at diagnosis were significantly higher for obese cancer patients compared with patients who were of normal weights $(\mathrm{P}=0.002$; Fig. 1). However, no significant difference was detected in median plasma HGF and adiponectin levels between normal, overweight and obese patients at baseline (Fig. 1). Notably, there were no significant correlations between the baseline levels of the evaluated adipokines (Table III).

Alterations in circulating adipokines following chemotherapy in patients with cancer. In order to investigate the changes in the blood profile levels of evaluated adipokines in patients with cancer following an adequate trial of chemotherapy, a second blood sample was collected after eight weeks of administration and then analyzed for the adipokines 
Table I. Demographic, anthropometric and basal adipokine characteristics of study groups $(\mathrm{n}=60)$.

\begin{tabular}{|c|c|c|c|}
\hline Variable & Healthy controls $(n=30)$ & Patients with cancer $(n=30)$ & P-value \\
\hline Age, years & $45(38.75-56.5)$ & $47(38.75-57.5)$ & 0.569 \\
\hline Waist, cm & $87(78-109)$ & $95(86.3-103.8)$ & 0.312 \\
\hline Waist-hip ratio & $0.91(0.82-0.99)$ & $0.91(0.85-0.96)$ & 0.886 \\
\hline BMI, $\mathrm{kg} / \mathrm{m}^{2}$ & $28.52(24.33-34.76)$ & $26.5(22.4-31.4)$ & 0.110 \\
\hline $\mathrm{HGF}, \mathrm{pg} / \mathrm{ml}$ & $729.98(620.13-886.90)$ & $721.66(558.43-850.59)$ & 0.894 \\
\hline Adiponectin, ng/ml & $5,152.03(3,364.09-7,293.30)$ & $5,695.63(39,94.04-7,680.45)$ & 0.340 \\
\hline Leptin, pg/ml & $25,349.74(21,355.73-29,139.97)$ & $22,741.41(20,622.14-29,669.79)$ & 0.679 \\
\hline Gender & & & 1.000 \\
\hline Male & $16(53.3)$ & $16(53.3)$ & \\
\hline Female & $14(46.7)$ & $14(46.7)$ & \\
\hline Marital status & & & 0.431 \\
\hline Single & $4(13.3)$ & $2(6.7)$ & \\
\hline Married & $26(86.7)$ & $27(90.0)$ & \\
\hline Divorced & $0(0.0)$ & $1(3.3)$ & \\
\hline Education & & & $0.001^{\mathrm{a}}$ \\
\hline Uneducated & $2(6.7)$ & $10(33.3)$ & \\
\hline High school & $17(56.7)$ & $8(26.7)$ & \\
\hline Diploma & $0(0.0)$ & $6(20.0)$ & \\
\hline College graduate & $11(36.7)$ & $6(20.0)$ & \\
\hline Smoking & & & $0.016^{\mathrm{a}}$ \\
\hline Never & $16(53.3)$ & $14(46.7)$ & \\
\hline Past & $1(3.3)$ & $9(30.0)$ & \\
\hline Current & $13(43.3)$ & $7(23.3)$ & \\
\hline $\begin{array}{l}\text { Family history of cancer } \\
\text { in first-degree relatives }\end{array}$ & & & $0.01^{\mathrm{a}}$ \\
\hline Present & $10(33.3)$ & $20(66.7)$ & \\
\hline Absent & $20(66.7)$ & $10(33.3)$ & \\
\hline
\end{tabular}

${ }^{\mathrm{a}} \mathrm{P}<0.05$. Data are presented as the median (25th-75th percentile) for continuous variables and as the frequency (\%) for categorical variables. BMI, body mass index; HGF, hepatocyte growth factor.

of interest. Notably, the plasma levels of all evaluated adipokines were increased following chemotherapy (Table IV). However, a significant alteration in the median plasma levels between baseline and follow-up was observed for adiponectin only $(\mathrm{P}=0.013)$. The median adiponectin plasma levels were significantly increased by $22.87 \%$ to $6,998.37 \mathrm{ng} / \mathrm{ml}$ (IQR, 4749.21-10,647.22) at follow-up among patients with cancer (Table IV). The median HGF and leptin plasma levels did not differ significantly between the baseline and follow-up samples $(\mathrm{P}=0.93$ and $\mathrm{P}=0.223$ for $\mathrm{HGF}$ and leptin levels, respectively; Table IV). The results of Spearman's correlation analysis revealed a significant positive correlation between baseline and follow-up adiponectin levels $(\rho=0.706 ; P=0.002)$. However, no significant correlation was detected between the levels of HGF and leptin at baseline and at follow-up among patients with cancer $(\rho=0.365, P=0.165$ for HGF; $\rho=0.144$, $\mathrm{P}=0.594$ for leptin). Further analysis revealed no significant correlation between the evaluated adipokines at follow-up. Subgroup analysis to examine whether the levels of adipokines at follow-up predicted the patient response to therapy was not performed due to the small sample size $(n=16)$.

\section{Discussion}

The current study assessed the circulating levels of selected adipokines in a series of patients newly diagnosed with solid malignancy, in order to define their correlation with tumor characteristics and measures of adiposity. The results demonstrated that the levels of circulating HGF, adiponectin and leptin included in the present study did not vary between patients with cancer and the healthy controls at the time of diagnosis. In addition, the evaluated adipokines did not correlate with each other at baseline or following chemotherapy among patients with cancer. The plasma levels of HGF were significantly higher in patients with advanced-stage disease; however, none of the circulating adipokines evaluated in the present study were associated with lymph node status. There was no significant difference in HGF and leptin levels between baseline and follow-up among patients with cancer. However, follow-up adiponectin levels differed significantly from the baseline levels, demonstrating a positive correlation. With the exception of leptin plasma levels, which exhibited a positive association with BMI, the evaluated adipokines did 
Table II. Adipokines and tumor characteristics of patients with cancer at baseline $(n=30)$.

\begin{tabular}{|c|c|c|c|c|c|c|}
\hline \multirow{2}{*}{$\begin{array}{l}\text { Tumor } \\
\text { characteristic }\end{array}$} & \multicolumn{2}{|c|}{ HGF, pg/ml } & \multicolumn{2}{|c|}{ Adiponectin, ng/ml } & \multicolumn{2}{|c|}{ Leptin, pg/ml } \\
\hline & Median & IQR & Median & IQR & Median & IQR \\
\hline \multicolumn{7}{|l|}{ Tumor type (no. of patients) } \\
\hline Colorectal (13) & 687.81 & $548.66-871.77$ & $5,695.63$ & $3,892.57-8,048.04$ & $21,192.70$ & $19,481.00-23,638.00$ \\
\hline Breast (10) & 775.57 & 638.06-912.65 & $5,792.86$ & $5,058.33-7,956.21$ & $30,240.40$ & $25,105.20-52,610.00$ \\
\hline Other (7) & 713.91 & $648.85-836.69$ & $4,954.11$ & 3,605.30-7,388.97 & $22,496.90$ & $19,888.50-2,9017.7$ \\
\hline P-value & & 0.840 & & 0.483 & & $0.004^{\mathrm{a}}$ \\
\hline \multicolumn{7}{|l|}{ Stage (no. of patients) } \\
\hline Early (I and II) (9) & 674.90 & $493.68-742.90$ & $5,210.12$ & $4,307.43-7,138.86$ & $29,506.80$ & $22,659.90-31055.50$ \\
\hline Advanced (III and IV) (21) & 797.30 & $628.40-958.67$ & $5,891.02$ & $3,838.95-7,702.83$ & $22,496.90$ & $19,888.50-26,735.40$ \\
\hline P-value & & $0.044^{\mathrm{a}}$ & & 0.803 & & 0.081 \\
\hline \multicolumn{7}{|l|}{$\begin{array}{l}\text { Lymph-node involvement } \\
\text { (no. of patients) }^{\mathrm{b}}\end{array}$} \\
\hline Positive (21) & 735.84 & 584.04-890.17 & $5,695.63$ & 3,838.95-7,332.77 & $22,822.90$ & $19,888.50-29,588.30$ \\
\hline Negative (7) & 697.00 & $499.00-756.40$ & $5,430.26$ & $4,602.96-8,582.09$ & $28,039.60$ & $22,659.90-30,974.00$ \\
\hline P-value & & 0.194 & & 0.614 & & 0.441 \\
\hline \multicolumn{7}{|l|}{$\begin{array}{l}\text { Surgical procedure } \\
\text { (no. of patients) }\end{array}$} \\
\hline Yes (24) & 704.81 & $546.31-799.56$ & $5,695.63$ & $4,112.60-7,562.70$ & $23,638.00$ & $20,948.20-30,770.20$ \\
\hline None (6) & 1016.43 & $697.65-1,640.32$ & $6,171.56$ & $3,766.51-8,603.78$ & $21,763.30$ & $19,847.80-23,352.70$ \\
\hline P-value & & $0.038^{\mathrm{a}}$ & & 0.959 & & 0.120 \\
\hline
\end{tabular}

${ }^{a} \mathrm{P}<0.05$ was considered statistically significant. ${ }^{\mathrm{b}}$ Two patients were missing from analysis. HGF, hepatocyte growth factor; IQR, interquartile range.
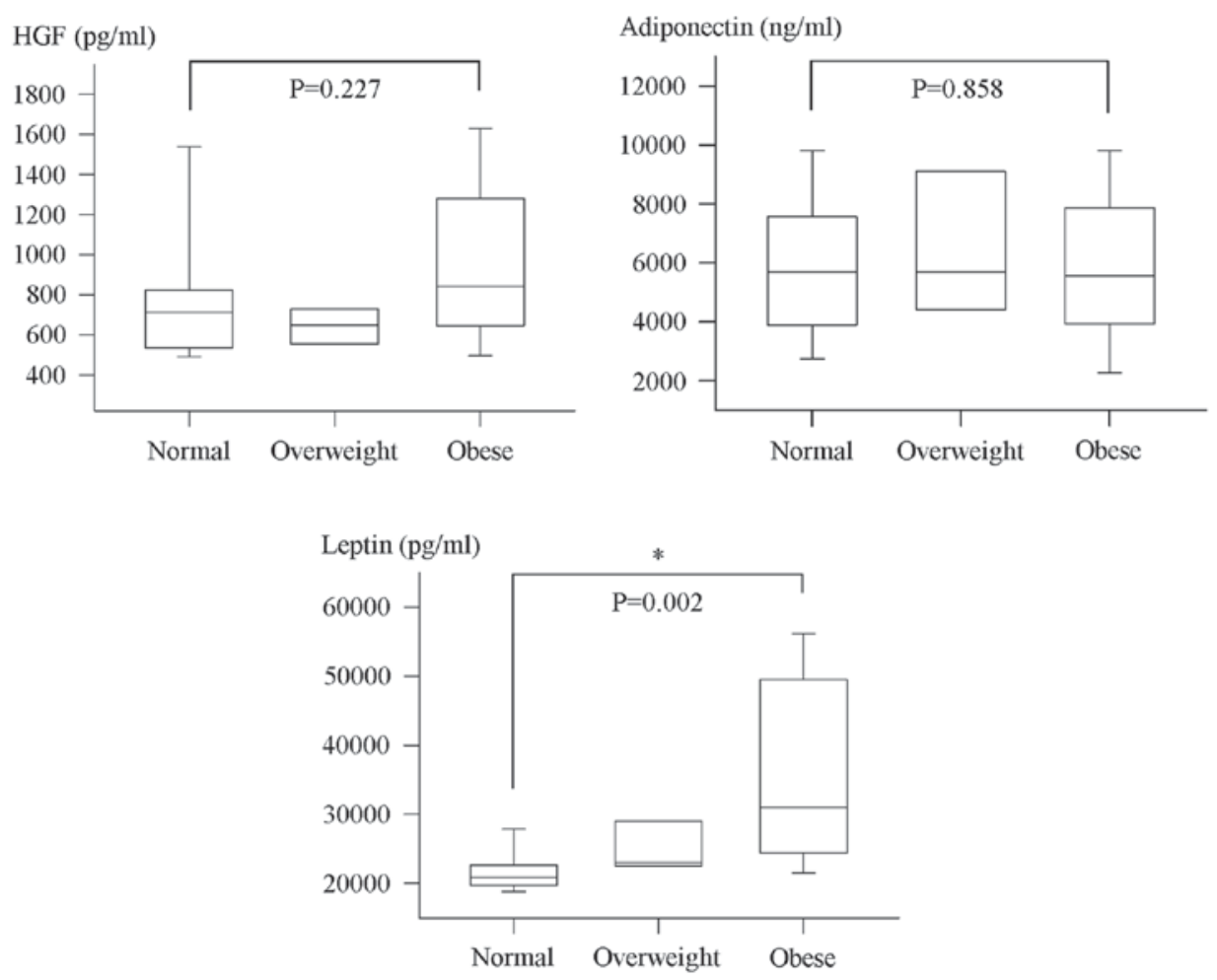

Figure 1. Baseline plasma levels of adipokines among patients with cancer according to BMI $(n=30)$. Boxplots represent median plasma levels of HGF, adiponectin and leptin among patients with cancer at the time of diagnosis, stratified based on BMI. The boxes represent the 25th and 75th percentiles, whereas the bars represent minimum and maximum values. The x-axis represents the groupings of patients with cancer according to BMI, and the $y$-axis represents plasma concentration of relative adipokines at the time of diagnosis. " $\mathrm{P}<0.05$, between BMI groups, according to Kruskal-Wallis analysis of variance. HGF, hepatocyte growth factor. BMI, body mass index. 
Table III. Correlation analysis of baseline adipokine levels with the anthropometric characteristics of patients with cancer ( $\mathrm{n}=30$ ).

\begin{tabular}{|c|c|c|c|c|c|c|}
\hline \multirow{2}{*}{$\begin{array}{l}\text { Anthropometric } \\
\text { characteristic }\end{array}$} & \multicolumn{2}{|c|}{$\mathrm{HGF}, \mathrm{pg} / \mathrm{ml}$} & \multicolumn{2}{|c|}{ Adiponectin, ng/ml } & \multicolumn{2}{|c|}{ Leptin, pg/ml } \\
\hline & $\rho$ & P-value & $\rho$ & P-value & $\rho$ & P-value \\
\hline Age, years & 0.432 & $0.017^{\mathrm{a}}$ & -0.144 & 0.447 & 0.079 & 0.680 \\
\hline $\mathrm{BMI}, \mathrm{kg} / \mathrm{m}^{2}$ & 0.072 & 0.704 & 0.019 & 0.921 & 0.657 & $<0.001^{\mathrm{a}}$ \\
\hline Waist, cm & -0.093 & 0.666 & -0.211 & 0.323 & 0.118 & 0.582 \\
\hline Waist-hip ratio & 0.164 & 0.443 & -0.182 & 0.394 & 0.194 & 0.363 \\
\hline $\mathrm{HGF}, \mathrm{pg} / \mathrm{ml}$ & - & - & 0.019 & 0.921 & -0.081 & 0.669 \\
\hline Adiponectin, ng/ml & 0.019 & 0.921 & - & - & 0.066 & 0.729 \\
\hline Leptin, $\mathrm{pg} / \mathrm{ml}$ & -0.081 & 0.669 & 0.066 & 0.729 & - & - \\
\hline
\end{tabular}

${ }^{a} \mathrm{P}<0.05$ was considered statistically significant. BMI, body mass index; HGF, hepatocyte growth factor; $\rho$, spearman's rank correlation coefficient.

Table IV. Variations in HGF, adiponectin and leptin titers following chemotherapy administration in patients with cancer ( $\mathrm{n}=16$ ).

\begin{tabular}{|c|c|c|c|c|c|c|}
\hline \multirow[b]{2}{*}{ Time point } & \multicolumn{2}{|c|}{ HGF, pg/ml } & \multicolumn{2}{|c|}{ Adiponectin, ng/ml } & \multicolumn{2}{|c|}{ Leptin, pg/ml } \\
\hline & Median & IQR & Median & IQR & Median & IQR \\
\hline Baseline & 721.66 & $558.43-850.59$ & 5695.63 & $3,994.04-7,680.45$ & $22,741.41$ & $20,622.14-29,669.79$ \\
\hline Follow-up & 829.12 & $651.92-1,219.27$ & 6998.37 & $4,749.21-10,647.22$ & $23,067.45$ & $20,255.34-29,343.75$ \\
\hline Percentage change vs. baseline & & 14.890 & & 22.870 & & 1.430 \\
\hline P-value & & 0.930 & & $0.013^{\mathrm{a}}$ & & 0.223 \\
\hline
\end{tabular}

${ }^{\mathrm{a} P}<0.05$ was considered statistically significant. HGF, hepatocyte growth factor; IQR, interquartile range.

not correlate significantly with anthropometric parameters or the measures of general and visceral obesity assessed in the current study.

The development and progression of cancer is hypothesized to be a complex and multi-factorial process (5). Obesity is a global health problem associated with a variety of metabolic diseases (24) and, at present, obesity is an established risk factor for epithelial tumors (25-27). Obesity is characterized as an excess of adipose tissue and defined by a BMI $\geq 30 \mathrm{~kg} / \mathrm{m}^{2}$ according to the WHO criteria (7). Additional measures of body fat include waist circumference and the waist-hip ratio, which are important for the evaluation of abdominal/visceral obesity, an additional marker of increased obesity-associated morbidity (20). Abdominal fat mass may vary considerably within a narrow range of total body fat and BMI (20). Previous studies focusing on populations that reside in the Middle East have determined cut-off points for waist circumference and waist-hip ratio that are similar to those suggested for Europeans $(20,28,29)$. According to the WHO, a waist circumference of $>94 \mathrm{~cm}$ in men and $>80 \mathrm{~cm}$ in women is associated with increased risk of metabolic complications (20). A waist-hip ratio of $\geq 0.90 \mathrm{~cm}$ in males and $\geq 0.85 \mathrm{~cm}$ in females is also associated with an increased risk of metabolic complications (20). Previous epidemiological and clinical data revealed that obesity is associated with increased rates of colorectal, breast, renal and endometrial cancer, as well as several other types of solid malignancy $(24,25)$. In addition, numerous studies have reported an association between visceral obesity and an increased risk of solid cancer and mortality (30-33). In the current study, baseline plasma adipokine levels measured in patients with cancer did not significantly differ from those of healthy controls. This finding suggests a lack of diagnostic potential for the adipokines HGF, adiponectin and leptin in this cohort of patients with solid cancer.

At present, it is well established that adipose tissue is a metabolically active endocrine organ that produces and secretes a wide range of hormones, cytokines and inflammatory molecules $(4,10,11,17,26,34)$. Adipokines refer to substances secreted from adipose tissue $(10,11,17,35)$ and the obese state alters the physiological functions of adipose tissue, leading to differing adipokine secretion and influencing the levels and functions of a variety of adipokines $(25,35)$. Adiponectin and leptin are abundant adipokines secreted by adipose tissue $(10,11,17,26)$. Leptin has been the focus of a number of studies as a potential mediator of obesity-associated cancer $(36,37)$. Leptin actions are mediated through the transmembrane leptin receptor, ObR (38-40). Signaling pathways activated by leptin include the cytokine Janus kinase/signal transducer and activator of transcription (STAT), phosphoinositide 3-kinase (PI3K) and mitogen-activated protein kinase (MAPK) signaling cascades $(11,38-41)$. In addition, leptin increases the expression levels of vascular endothelial growth factor, promoting endothelial cell proliferation and migration (42). Collectively, these signaling pathways increase 
cancer cell proliferation, motility and overall cancer progression (25). Previous studies revealed that circulating leptin levels positively correlate with adiposity $(11,38)$. Concordant with these findings, the results of the current study revealed that plasma leptin levels were positively correlated with BMI among patients with cancer. However, leptin levels did not exhibit a significant correlation with measured parameters of visceral obesity (waist circumference and waist-hip ratio). Although leptin levels are increased in the obese state, clinical studies providing correlations between circulating leptin levels and risk or prognosis among patients with cancer revealed conflicting results (43-46). In the present study, median plasma leptin levels exhibited differences based on tumor type. This suggests that leptin may serve as a potential prognostic or predictive marker for certain types of tumor, but not for others. However, leptin levels were not associated with tumor stage or lymph node status in the present study.

In human serum, adiponectin exists as a low molecular weight trimer, intermediate molecular weight hexamer and high molecular weight multimer (47). Adiponectin activity is mediated through its seven transmembrane receptors, including AdipoR1 and AdipoR2 (48). Adiponectin functions via the 5 ' adenosine monophosphate-activated protein kinase (AMPK), mammalian target of rapamycin (mTOR), PI3K/protein kinase B (Akt), MAPK, STAT, nuclear factor $\kappa \mathrm{B}(\mathrm{NF}-\mathrm{\kappa B})$ and the sphingolipid metabolic signaling pathways $(11,47)$. At present, the results from certain studies support the hypothesis of a protective effect for adiponectin in cancer, through its antiproliferative and apoptosis-inducing activities $(49,50)$. Previous studies have revealed that plasma adiponectin concentrations correlate inversely with BMI (50-52). However, in the present study, the results did not reveal a significant association between plasma levels of adiponectin and BMI among patients with cancer. Although adiponectin plasma levels were inversely correlated with measures of visceral obesity, this correlation did not reach statistical significance in the present study. In addition, there were no significant correlations between adiponectin plasma levels and the assessed tumor characteristics in patients. However, adiponectin was the only adipokine in the current study that exhibited a significant alteration in patients with cancer, following the adequate administration of chemotherapy. Despite the low number of subjects in the present study, these findings may suggest that adiponectin is a potential adipokine to evaluate during the follow-up of patients with cancer, in order to further investigate the correlation between its plasma levels and patient response to treatment.

Although a number of studies have focused on key adipokines, such as adiponectin and leptin $(18,53)$, a number of other candidate adipokines have been investigated with regard to adiposity and cancer $(12,54)$. The results of previous studies revealed that HGF is expressed and secreted by adipocytes, qualifying it as an adipokine $(9,17,55)$. HGF is an angiogenic growth factor that, when bound to its receptor c-Met, promotes cancer cell proliferation, migration, invasion and metastasis in numerous types of solid human tumors $(56,57)$. The biological functions of the HGF/c-Met axis are mediated through a variety of downstream effectors, including the RAS-MAPK and PI3K/Akt/NF-kB signaling pathways $(58,59)$. Circulating HGF levels have been observed to be elevated in obese individuals, compared with those in individuals of a normal body weight $(9,26,34,60)$. In the present study, no association was detected between HGF levels and BMI or measures of visceral obesity in patients with cancer. However, Faber et al (61) revealed that visceral adipose tissue was significantly associated with circulating levels of HGF, irrespective of BMI. The current study demonstrated that increased HGF levels were associated with advanced age among patients, and plasma HGF levels were significantly higher in patients with cancer with advanced-stage disease, compared with patients presenting with early-stage disease. In this regard, the circulating levels of HGF may be considered to evaluate the grade of malignancy and degree of invasiveness, particularly in patients whose disease state is not able to be determined effectively with the use of more reliable blood markers. The results of the present study revealed no correlation between the plasma levels of HGF and those of leptin and adiponectin among patients with cancer.

In the present study, the interval between plasma samples was relatively long (eight weeks). Although this time point may provide a sufficient duration to detect alterations in circulating adipokine levels, it is possible that early changes in adipokine levels may have been missed in the current study. However, a marked change in the plasma concentration of adiponectin was detected in certain patients. This primary finding may suggest that adiponectin levels are the most sensitive to chemotherapy; therefore, further investigations are required to assess the prognostic and predictive potential of this adipokine.

Although, to the best of our knowledge, this study is the first of its type among Jordanian patients with cancer, previous evaluations of the prognostic and predictive roles of circulating adipokines have been conducted. Karapanagiotou et al (62) investigated the significance of serum adipokines as diagnostic and prognostic markers in patients with advanced non-small cell lung cancer (NSCLC). Data from the current study are concordant with the results obtained by this previous study, which revealed that serum leptin and adiponectin levels at the time of diagnosis did not differ significantly between patients with NSCLC and healthy controls. Although baseline serum leptin levels were significantly associated with increased BMI among patients with lung cancer, adiponectin levels lacked a significant correlation with BMI (62), concordant with the data from the current study. Additionally, consistent with the results of the current study, Karapanagiotou et al (62) also reported increased serum levels of leptin and adiponectin following an adequate trial of chemotherapy. Although beyond the scope of this study, Karapanagiotou et al (62) revealed a lack of prognostic value for the assessed adipokines in patients with advanced NSCLC. Concordant with these findings, a recent study by Slomian et al (63) revealed a significant increase in circulating levels of leptin and adiponectin in a cohort of patients with advanced colorectal cancer who required palliative chemotherapy.

The current study had certain limitations, including the low number of patients enrolled and the lack of follow-up blood sampling. The limited sample size available hindered further subgroup analysis and sample stratification. In addition, due to the relatively small sample size of the present study, particular 
associations may not have reached the required significance level.

In conclusion, the circulating levels of a number of adipokines have been previously investigated in patients with cancer; however, the current study provides additional insights into the variations in adipokine levels over time, their intercorrelations and the associations of these adipokines with general measures of adiposity in patients with cancer. To the best of our knowledge, this is the first study to assess the correlations between circulating adipokine levels and disease characteristics and adiposity measures among Jordanian patients with cancer at the time of diagnosis. The findings in the current study revealed no correlation between patient HGF, adiponectin and leptin plasma levels at the time of diagnosis or following chemotherapy. Circulating leptin levels were positively associated with BMI. However, none of the measured adipokines were associated with visceral obesity, as determined by waist circumference and waist-hip ratio. Plasma adipokine levels increased following chemotherapy administration; however the levels of adiponectin may be the most reliably altered, and this adipokine requires further evaluation for its potential role as a prognostic marker. In addition, further studies are required to clarify the associations between adipokines and solid tumor burden, and examine how adipokine levels are altered upon the administration of systemic therapy. The use of circulating adipokines as markers to stratify patients and monitor responses to therapy may warrant further investigation. Evaluation of specific adipokine levels may be useful in epidemiological studies, and may improve the methods of characterizing disease risk and response to therapy among overweight and obese individuals.

\section{Acknowledgements}

The current study was supported by the Deanship of Research at Jordan University of Science and Technology (grant no. 20140057). The authors would like to thank the patients who contributed blood samples for this study and the hospital staff for assistance with sample collection.

\section{References}

1. Siegel RL, Miller KD and Jemal A: Cancer statistics, 2016. CA Cancer J Clin 66: 7-30, 2016.

2. World Health Organization (WHO): Cancer Fact Sheet 2015 http://www.who.int/mediacentre/factsheets/fs297/en/. Accessed July 2, 2016.

3. Jordan Ministry of Health (MOH). National Cancer Registry 2012. file:///C:/Users/ws/Downloads/annual\%20report-2012.pdf Accessed June 23, 2016.

4. Ceschi M, Gutzwiller F, Moch H, Eichholzer M and Probst-Hensch NM: Epidemiology and pathophysiology of obesity as cause of cancer. Swiss Med Wkly 137: 50-56, 2007.

5. Davoodi SH, Malek-Shahabi T, Malekshahi-Moghadam A, Shahbazi R and Esmaeili S: Obesity as an important risk factor for certain types of cancer. Iran J Cancer Prev 6: 186-194, 2013.

6. World Health Organization (WHO). Obesity and overweight Fact Sheet 2016. http://www.who.int/mediacentre/factsheets/fs311/en/. Accessed on July 2, 2016.

7. Obesity: Preventing and managing the global epidemic. Report of a WHO Consultation. World Health Organ Tech Rep Ser: 894: i-xii, 1-253, 2000.

8. Hillon P, Guiu B, Vincent J and Petit JM: Obesity, type 2 diabetes and risk of digestive cancer. Gastroenterol Clin Biol 34: 529-533, 2010.
9. Lin JH, Gunter MJ, Manson JE, Rexrode KM, Cook NR, Kraft P, Cochrane BB, Chlebowski RT, Ho GY and Zhang SM: The aromatase gene (CYP19A1) variants and circulating hepatocyte growth factor in postmenopausal women. PloS One 7: e42079, 2012.

10. Fain JN, Madan AK, Hiler ML, Cheema P and Bahouth SW: Comparison of the release of adipokines by adipose tissue, adipose tissue matrix and adipocytes from visceral and subcutaneous abdominal adipose tissues of obese humans. Endocrinology 145: 2273-2282, 2004.

11. Vucenik I and Stains JP: Obesity and cancer risk: Evidence, mechanisms and recommendations. Ann N Y Acad Sci 1271: 37-43, 2012.

12. Assiri AM and Kamel HF: Evaluation of diagnostic and predictive value of serum adipokines: Leptin, resistin and visfatin in postmenopausal breast cancer. Obes Res Clin Pract 10: 442-453, 2016.

13. Alshaker H, Sacco K, Alfraidi A, Muhammad A, Winkler M and Pchejetski D: Leptin signalling, obesity and prostate cancer: Molecular and clinical perspective on the old dilemma. Oncotarget 6: 35556-35563, 2015.

14. Lin T, Zhao X and Kong WM: Association between adiponectin levels and endometrial carcinoma risk: Evidence from a dose-response meta-analysis. BMJ Open 5: e008541, 2015.

15. Zhang YW, Su Y, Volpert OV and Vande Woude GF: Hepatocyte growth factor/scatter factor mediates angiogenesis through positive VEGF and negative thrombospondin 1 regulation. Proc Natl Acad Sci USA 100: 12718-12723, 2003.

16. Scagliotti GV, Novello S and von Pawel J: The emerging role of MET/HGF inhibitors in oncology. Cancer Treat Rev 39: 793-801, 2013.

17. Faber DR, Moll FL, Vink A, van der Waal C, Kalkhoven E, Schipper HS, Hajer GR, Monajemi H and Visseren FL: Adipose tissue quantity and composition contribute to adipokine concentrations in the subclavian vein and the inferior mesenteric vein. Int J Obes (Lond) 36: 1078-1085, 2012.

18. Hefetz-Sela S and Scherer PE: Adipocytes: Impact on tumor growth and potential sites for therapeutic intervention. Pharmacol Ther 138: 197-210, 2013.

19. Edge S, Byrd DR, Compton CC, Fritz AG, Greene FL and Trotti A: AJCC cancer staging handbook from the AJCC cancer staging manual. 7th edition, 2010.

20. World HealthOrganization (WHO): Circumference and Waist-Hip Ratio: Report of a WHO Expert Consultation. Geneva, 8-11, 2008.

21. Eisenhauer EA, Therasse P, Bogaerts J, Schwartz LH, Sargent D, Ford R, Dancey J, Arbuck S, Gwyther S, Mooney M, et al: New response evaluation criteria in solid tumours: Revised RECIST guideline (version 1.1). Eur J Cancer 45: 228-247, 2009.

22. Bonen A, Campbell SE, Benton CR, Chabowski A, Coort SL, Han XX, Koonen DP, Glatz JF and Luiken JJ: Regulation of fatty acid transport by fatty acid translocase/CD36. Proc Nutr Soc 63: 245-249, 2004.

23. Hong Y, Davis B, Marron JS, Kwitt R, Singh N, Kimbell JS, Pitkin E, Superfine R, Davis SD, Zdanski CJ and Niethammer M: Statistical atlas construction via weighted functional boxplots. Med Image Anal 18: 684-698, 2014.

24. Dubois V, Delort L, Billard H, Vasson MP and Caldefie-Chezet F: Breast cancer and obesity: In vitro interferences between adipokines and proangiogenic features and/or antitumor therapies? PloS One 8: e58541, 2013.

25. Strong AL, Burow ME, Gimble JM and Bunnell BA: Concise review: The obesity cancer paradigm: Exploration of the interactions and crosstalk with adipose stem cells. Stem Cells 33: 318-326, 2015.

26. Silha JV, Krsek M, Sucharda P and Murphy LJ: Angiogenic factors are elevated in overweight and obese individuals. Int J Obes (Lond) 29: 1308-1314, 2005.

27. Moore T, Beltran L, Carbajal S, Hursting SD and DiGiovanni J: Energy balance modulates mouse skin tumor promotion through altered IGF-1R and EGFR crosstalk. Cancer Prev Res (Phila) 5: 1236-1246, 2012

28. Lear SA, James PT, Ko GT and Kumanyika S: Appropriateness of waist circumference and waist-to-hip ratio cutoffs for different ethnic groups. Eur J Clin Nutr 64: 42-61, 2010.

29. Alberti KG, Eckel RH, Grundy SM, Zimmet PZ, Cleeman JI, Donato KA, Fruchart JC, James WP, Loria CM, Smith SC Jr, et al: Harmonizing the metabolic syndrome: A joint interim statement of the international diabetes federation task force on epidemiology and prevention; National heart, lung and blood institute; American heart association; World heart federation; International atherosclerosis society; and international association for the study of obesity. Circulation 120: 1640-1645, 2009. 
30. Moghaddam AA, Woodward M and Huxley R: Obesity and risk of colorectal cancer: A meta-analysis of 31 studies with 70,000 events. Cancer Epidemiol Biomarkers Prev 16: 2533-2547, 2007.

31. Harvie M, Hooper L and Howell AH: Central obesity and breast cancer risk: A systematic review. Obes Rev 4: 157-173, 2003.

32. Wierup I, Carlsson AC, Wündell P, Riserus U, Ärnlöv J and Borné Y: Low anthropometric measures and mortality-results from the Malmö Diet and cancer study. Ann Med 47: 325-331, 2015.

33. Park SW, Lee HL, Doo EY, Lee KN, Jun DW, Lee OY, Han DS, Yoon BC, Choi HS and Lee KH: Visceral obesity predicts fewer lymph node metastases and better overall survival in colon cancer. J Gastrointest Surg 19: 1513-1521, 2015.

34. Lee SA, Kallianpur A, Xiang YB, Wen W, Cai Q, Liu D, Fazio S, Linton MF, Zheng W and Shu XO: Intra-individual variation of plasma adipokine levels and utility of single measurement of these biomarkers in population-based studies. Cancer Epidemio Biomarkers Prev 16: 2464-2470, 2007.

35. Ayoub NM and Kaddoumi A: Obesity and breast cancer: Molecular and epidemiological evidence. Journal of Cancer Research Updates 4: 30-42, 2015.

36. Giordano C, Vizza D, Panza S, Barone I, Bonofiglio D, Lanzino M, Sisci D, De Amicis F, Fuqua SA, Catalano S and Andò S: Leptin increases HER2 protein levels through a STAT3-mediated up-regulation of Hsp90 in breast cancer cells. Mol Oncol 7: 379-391, 2013.

37. Huang CY, Yu HS, Lai TY, Yeh YL, Su CC, Hsu HH, Tsai FJ, Tsai $\mathrm{CH}, \mathrm{Wu} \mathrm{HC}$ and Tang $\mathrm{CH}$ : Leptin increases motility and integrin up-regulation in human prostate cancer cells. J Cell Physiol 226: 1274-1282, 2011.

38. Fiorio E, Mercanti A, Terrasi M, Micciolo R, Remo A, Auriemma A, Molino A, Parolin V, Di Stefano B, Bonetti F, et al: Leptin/HER 2 crosstalk in breast cancer: In vitro study and preliminary in vivo analysis. BMC Cancer 8: 305, 2008.

39. Alegre MM, Knowles MH, Robison RA and O'Neill KL: Mechanics behind breast cancer prevention-focus on obesity, exercise and dietary fat. Asian Pac J Cancer Prev 14: 2207-2212, 2013.

40. Ray A, Nkhata KJ and Cleary MP: Effects of leptin on human breast cancer cell lines in relationship to estrogen receptor and HER2 status. Int J Oncol 30: 1499-1509, 2007.

41. Wang D, Chen J, Chen H, Duan Z, Xu Q, Wei M, Wang L and Zhong $\mathrm{M}$ : Leptin regulates proliferation and apoptosis of colorectal carcinoma through $\mathrm{PI} 3 \mathrm{~K} / \mathrm{Akt} / \mathrm{mTOR}$ signalling pathway. J Biosci 37: 91-101, 2012

42. Terrasi M, Bazan V, Caruso S, Insalaco L, Amodeo V, Fanale D, Corsini LR, Contaldo C, Mercanti A, Fiorio E, et al: Effects of PPAR $\gamma$ agonists on the expression of leptin and vascular endothelial growth factor in breast cancer cells. J Cell Physiol 228: 1368-1374, 2013

43. Du J, Han JC, Zhang YJ, Qi GB, Zhang Y and Li HB: Relationship between serum leptin levels and non-small cell lung carcinoma: A meta-analysis. Genet Mol Res 14: 13699-13708, 2015.

44. Grossmann ME and Cleary MP: The balance between leptin and adiponectin in the control of carcinogenesis-focus on mammary tumorigenesis. Biochimie 94: 2164-2171, 2012

45. Stolzenberg-Solomon RZ, Newton CC, Silverman DT, Pollak M, Nogueira LM, Weinstein SJ, Albanes D, Männistö S and Jacobs EJ: Circulating leptin and risk of pancreatic cancer: A pooled analysis from 3 cohorts. Am J Epidemiol 182: 187-197, 2015.
46. Gong TT, Wu QJ, Wang YL and Ma XX: Circulating adiponectin, leptin and adiponectin-leptin ratio and endometrial cancer risk: Evidence from a meta-analysis of epidemiologic studies. Int J Cancer 137: 1967-1978, 2015.

47. Obeid $\mathrm{S}$ and Hebbard L: Role of adiponectin and its receptors in cancer. Cancer Biol Med 9: 213-220, 2012.

48. Shin E, Yu YD, Kim DS and Won NH: Adiponectin receptor expression predicts favorable prognosis in cases of hepatocellular carcinoma. Pathol Oncol Res 20: 667-675, 2014.

49. Dalamaga M, Diakopoulos KN and Mantzoros CS: The role of adiponectin in cancer: A review of current evidence. Endocr Rev 33: 547-594, 2012.

50. Delort L, Jardé T, Dubois V, Vasson MP and Caldefie-Chezet F: New insights into anticarcinogenic properties of adiponectin: A potential therapeutic approach in breast cancer? Vitam Horm 90: 397-417, 2012.

51. Vona-Davis L and Rose DP: Adipokines as endocrine, paracrine, and autocrine factors in breast cancer risk and progression. Endocr Relat Cancer 14: 189-206, 2007.

52. Otvos L Jr, Haspinger E, La Russa F, Maspero F, Graziano P, Kovalszky I, Lovas S, Nama K, Hoffmann R, Knappe D, et al: Design and development of a peptide-based adiponectin receptor agonist for cancer treatment. BMC Biotechnol 11: 90, 2011.

53. Lee $\mathrm{CH}$, Woo YC, Wang Y, Yeung CY, Xu A and Lam KS: Obesity, adipokines and cancer: An update. Clin Endocrinol (Oxf) 83: 147-156, 2015

54. Dalamaga M: Obesity, insulin resistance, adipocytokines and breast cancer: New biomarkers and attractive therapeutic targets. World J Exp Med 3: 34-42, 2013.

55. Rehman J, Traktuev D, Li J, Merfeld-Clauss S, Temm-Grove CJ, Bovenkerk JE, Pell CL, Johnstone BH, Considine RV and March KL: Secretion of angiogenic and antiapoptotic factors by human adipose stromal cells. Circulation 109: 1292-1298, 2004.

56. Stein GY, Yosef N, Reichman H, Horev J, Laser-Azogui A, Berens A, Resau J, Ruppin E, Sharan R and Tsarfaty I: Met kinetic signature derived from the response to HGF/SF in a cellular model predicts breast cancer patient survival. PloS One 7: e45969, 2012

57. Tang Z, Du R, Jiang S, Wu C, Barkauskas DS, Richey J, Molter J, Lam M,Flask C, Gerson S, et al: Dual MET-EGFR combinatorial inhibition against T790M-EGFR-mediated erlotinib-resistant lung cancer. Br J Cancer 99: 911-922, 2008.

58. Sattler M and Salgia R: The MET axis as a therapeutic target. Update Cancer Ther 3: 109-118, 2009.

59. Lawrence RE and Salgia R: MET molecular mechanisms and therapies in lung cancer. Cell Adh Migr 4: 146-152, 2010.

60. Rehman J, Considine RV, Bovenkerk JE, Li J, Slavens CA, Jones RM and March KL: Obesity is associated with increased levels of circulating hepatocyte growth factor. J Am Coll Cardiol 41: 1408-1413, 2003.

61. Faber DR, van der Graaf Y, Westerink J, Kanhai DA, Monajemi H and Visseren FL; SMART study Group: Hepatocyte growth factor and interferon- $\gamma$ inducible protein-10 are related to visceral adiposity. Eur J Clin Invest 43: 369-378, 2013.

62. Karapanagiotou EM, Tsochatzis EA, Dilana KD, Tourkantonis I, Gratsias I and Syrigos KN: The significance of leptin, adiponectin, and resistin serum levels in non-small cell lung cancer (NSCLC). Lung cancer 61: 391-397, 2008.

63. Slomian G, Swietochowska E, Malinowska-Borowska J, Kasperczyk S, Rogalska A and Nowak P: Association between chemotherapy and plasma adipokines in patients with colorectal cancer. Pharmacol Rep 66: 902-907, 2014. 